\title{
SCIENTIFIC REP RTS \\ Expression of the POTE gene family in human ovarian cancer
}

Received: 5 July 2018

Accepted: 7 November 2018

Published online: 20 November 2018

\author{
Carter J Barger ${ }^{1,2}$, Wa Zhang ${ }^{1,2}$, Ashok Sharma $\mathbb{1}^{1,2}$, Linda Chee ${ }^{1,2}$, Smitha R. James ${ }^{3}$, \\ Christina N. Kufel ${ }^{3}$, Austin Miller $\mathbb{1}^{4}$, Jane Meza ${ }^{5}$, Ronny Drapkin $\mathbb{1}^{6}{ }^{6}$, Kunle Odunsi ${ }^{7,8,9}$, \\ David Klinkebiel ${ }^{2,10}$ \& Adam R. Karpf ${ }^{1,2,3}$
}

The POTE family includes 14 genes in three phylogenetic groups. We determined POTE mRNA expression in normal tissues, epithelial ovarian and high-grade serous ovarian cancer (EOC, HGSC), and pan-cancer, and determined the relationship of POTE expression to ovarian cancer clinicopathology. Groups 1 \& 2 POTEs showed testis-specific expression in normal tissues, consistent with assignment as cancer-testis antigens (CTAs), while Group 3 POTEs were expressed in several normal tissues, indicating they are not CTAs. Pan-POTE and individual POTEs showed significantly elevated expression in EOC and HGSC compared to normal controls. Pan-POTE correlated with increased stage, grade, and the HGSC subtype. Select individual POTEs showed increased expression in recurrent HGSC, and $P O T E E$ specifically associated with reduced HGSC OS. Consistent with tumors, EOC cell lines had significantly elevated Pan-POTE compared to OSE and FTE cells. Notably, Group 1 \& 2 POTEs (POTEs $A / B / B 2 / C / D)$, Group 3 POTE-actin genes (POTEs E/F/I/J/KP), and other Group 3 POTEs (POTEs G/H/M) show within-group correlated expression, and pan-cancer analyses of tumors and cell lines confirmed this relationship. Based on their restricted expression in normal tissues and increased expression and association with poor prognosis in ovarian cancer, POTEs are potential oncogenes and therapeutic targets in this malignancy.

Epithelial ovarian cancer (EOC) is the most lethal gynecologic malignancy, and high-grade serous cancer (HGSC) is the most prevalent EOC subtype ${ }^{1,2}$. The majority of HGSC cases are diagnosed at late clinical stages. Once diagnosed, EOC and HGSC treatment consists of primary debulking surgery and platinum/taxane combination chemotherapy, typically leading to a robust clinical response. Unfortunately, most patients diagnosed in late stage ultimately relapse with chemoresistant disease ${ }^{3}$. Although there has been significant recent progress in ovarian cancer treatment ${ }^{4-6}$, there remains an urgent need for improved therapeutic approaches, particularly in the recurrent disease setting.

Cancer-testis antigens (CTAs), also known as cancer-germline genes, show low expression in normal somatic tissues but are expressed in germ cells of the adult testis and fetal ovary, and in placenta ${ }^{7,8}$. CTAs can show highly elevated expression in cancer, which appears most often to result from epigenetic alterations, particularly DNA hypomethylation ${ }^{9-11}$. Some CTAs are immunogenic (hence the name), in part because their normal expression is restricted to immune privileged sites. The immunogenicity of specific CTAs has led to the development of immunotherapies to target them in cancer, using vaccines and adoptive cell therapies ${ }^{8,9}$. Importantly, specific CTAs directly promote oncogenic phenotypes, suggesting they are not just cancer passengers ${ }^{12-14}$. This opens up new opportunities for therapeutic targeting of CTAs unrelated to immunotherapy, which is a crucial development, as only a limited number of CTAs are likely to be immunogenic.

\footnotetext{
${ }^{1}$ Eppley Institute for Cancer Research, University of Nebraska Medical Center, Omaha, NE, 68198-6805, USA. ${ }^{2}$ Fred \& Pamela Buffett Cancer Center, University of Nebraska Medical Center, Omaha, NE, 68198-6805, USA. ${ }^{3}$ Department of Pharmacology and Therapeutics, Roswell Park Comprehensive Cancer Center, Buffalo, NY, 14263, USA. ${ }^{4}$ Department of Biostatistics, Roswell Park Comprehensive Cancer Center, Buffalo, NY, 14263, USA. ${ }^{5}$ Department of Biostatistics, University of Nebraska Medical Center, Omaha, NE, 68198-4375, USA. ${ }^{\circ}$ Penn Ovarian Cancer Research Center, University of Pennsylvania Perelman School of Medicine, Philadelphia, PA, 19104, USA. ${ }^{7}$ Department of Immunology, Roswell Park Comprehensive Cancer Center, Buffalo, NY, 14263, USA. ${ }^{8}$ Department of Gynecologic Oncology, Roswell Park Comprehensive Cancer Center, Buffalo, NY, 14263, USA. ${ }^{9}$ Center for Immunotherapy, Roswell Park Comprehensive Cancer Center, Buffalo, NY, 14263, USA. ${ }^{10}$ Department of Biochemistry, University of Nebraska Medical Center, Omaha, NE, 68198, USA. Carter J Barger and Wa Zhang contributed equally. Correspondence and requests for materials should be addressed to A.R.K. (email: adam.karpf@unmc.edu)
} 


\begin{tabular}{|c|c|c|c|c|}
\hline HUGO name & Original name ${ }^{a}$ & Group $^{b}$ & Actin fusion & Testis-specific \\
\hline POTEA & POTE8 & 1 & - & $\sqrt{ }$ \\
\hline POTEB & POTE15 & 2 & - & $\sqrt{ }$ \\
\hline POTEB2 & $n / a$ & 2 & - & $\sqrt{ }$ \\
\hline POTEB $^{\mathrm{d}}$ & $n / a$ & 2 & - & $\mathrm{n} / \mathrm{d}$ \\
\hline POTEC & POTE18 & 2 & - & $\sqrt{ }$ \\
\hline POTED & POTE21 & 2 & - & $\sqrt{ }$ \\
\hline POTEE & POTE2 $\gamma$ & 3 & $\sqrt{ }$ & - \\
\hline POTEF & POTE $2 \alpha$ & 3 & $\sqrt{ }$ & - \\
\hline POTEG & POTE14 $\alpha$ & 3 & - & - \\
\hline POTEH & POTE22 & 3 & - & - \\
\hline POTEI & POTE $2 \beta^{\prime}$ & 3 & $\sqrt{ }$ & - \\
\hline POTEJ & POTE $2 \beta$ & 3 & $\sqrt{ }$ & - \\
\hline POTEKP & POTE2 $\delta$ & 3 & $\sqrt{ }$ & - \\
\hline POTEM & POTE14 $\beta$ & 3 & - & - \\
\hline
\end{tabular}

Table 1. Human POTE Gene Family. n/a: not applicable; n/d: not determined. ${ }^{a}$ Corresponds to chromosomal location; Bera et al., PNAS, 2002. 'based on phylogeny; Hahn et al., Gene, 2006, 238-245. ' GTEx RNAseq data;

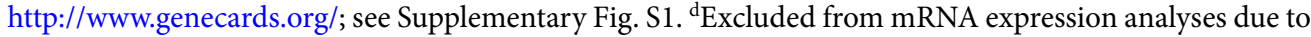
insufficient data.

A sizable number of CTAs, including the most frequently studied members of this superfamily, are located on the X-chromosome (CT-X genes). However, most CTAs were recently shown to be encoded on autosomes ${ }^{13,15}$. Amongst these, POTEs are the only multigene family described to date, POTEs consist of 14 primate-specific genes distributed on seven chromosomes, and are divided into three phylogenetic groups ${ }^{16-18}$. The POTE family originated from an ancestral ankyrin repeat domain 26 (ANKRD26) gene ${ }^{17}$. POTEs contain a conserved $3^{\prime} \mathrm{UTR}$ LINE-1 element, which promoted POTE dispersal in the primate genome, and several Chr. 2 POTEs contain a C-terminal in-frame fusion with Actin resulting from transposition ${ }^{16,19}$ (Table 1). Structurally, POTE proteins contain a N-terminal cysteine-rich region, central ankyrin repeats, and C-terminal spectrin-like $\alpha$-helices, suggesting participation in protein-protein interactions and association with cell membranes ${ }^{19,20}$.

An important early study of POTE expression in cancer showed differential POTE expression in cancer tissues, including ovarian cancer. However, the analysis of ovarian cancer was limited to an endpoint RT-PCR study of five ovarian cancer samples of unknown classification ${ }^{21}$. A limitation to early studies of POTEs was that the high homology of POTEs made it difficult to resolve expression of individual POTEs. However, in recent years, the field has experienced the advent of RNA-sequencing (RNA-seq), which can readily resolve individual POTEs, as well as great progress by consortia-based projects for depositing extensive RNA-seq data from normal human tissues, human tumors, and human cancer cell lines ${ }^{2,22-24}$. These data allow the opportunity to measure POTE expression in different contexts, including ovarian cancer. Here we report several new and extensive analyses of POTE expression, including in normal tissues, ovarian cancer tumors and cell lines, normal control cells, and an initial study in pan-cancer tissues and cell lines.

\section{Results}

POTE expression in normal human tissues. We first analyzed expression of $13 / 14$ members of the POTE gene family (data was not available for POTEB3) (Table 1), using GTEx RNAseq data ${ }^{22}$, primarily to determine if POTEs show a testis-specific or testis-enriched expression characteristic of CTAs ${ }^{7}$. Notably, Groups 1 \& 2 POTEs, which are more closely related to the ancestral ANKRD26 gene ${ }^{17}$, displayed testis-specific expression (Supplementary Fig. S1), despite the fact that ANKRD26 was widely expressed in normal tissues (data not shown). In contrast to Group 1 \& 2 POTEs, Group 3, and particularly the POTE-actin genes, showed widespread normal tissue expression (Supplementary Fig. S1). The only exception was POTEH, a Group 3 POTE that showed significant expression only in testis and prostate. We conclude that Groups $1 \& 2$ POTEs $(A, B, B 2, C, D)$ have normal tissue expression consistent with CTAs, while Group 3 POTEs (E, F, G, H, I, J, KP, M) do not (Table 1). Widespread expression of POTE-actin genes suggests a function in normal tissues.

POTE expression in EOC. We measured Pan-POTE expression by RT-qPCR in EOC and bulk normal ovary (NO) tissues. Supplementary Table S1 lists the characteristics of the EOC samples. Pan-POTE was significantly overexpressed in EOC compared to NO, with approximately one-third of cases showing $>10$-fold increased expression (Fig. 1a). Pan-POTE expression significantly associated with increased clinical stage and pathological grade (Fig. 1b,c). We separated EOC into HGSC (serous histology, grade 2/3) and other EOC. While Pan-POTE was elevated in both groups compared to NO, HGSC showed significantly higher expression (Fig. 1d). Individual histological subgroups did not contain sufficient samples to make meaningful comparisons (Supplementary Fig. S2). Next, to assess individual POTE gene expression in EOC, we used Affymetrix microarrays to examine EOC $(n=40)$ and $\mathrm{NO}(\mathrm{n}=3)$. In agreement with Pan-POTE data, sub-sets of POTEs showed elevated expression in EOC (Supplementary Fig. S3). However, this methodology was limited by extensive POTE gene overlap. 
a

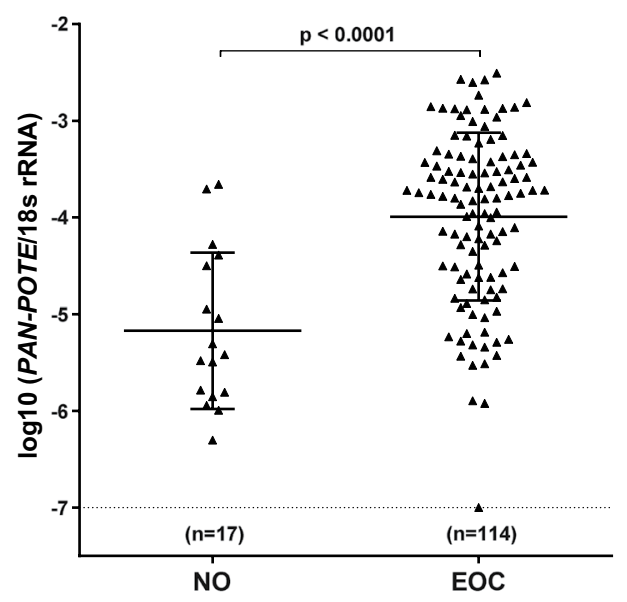

C

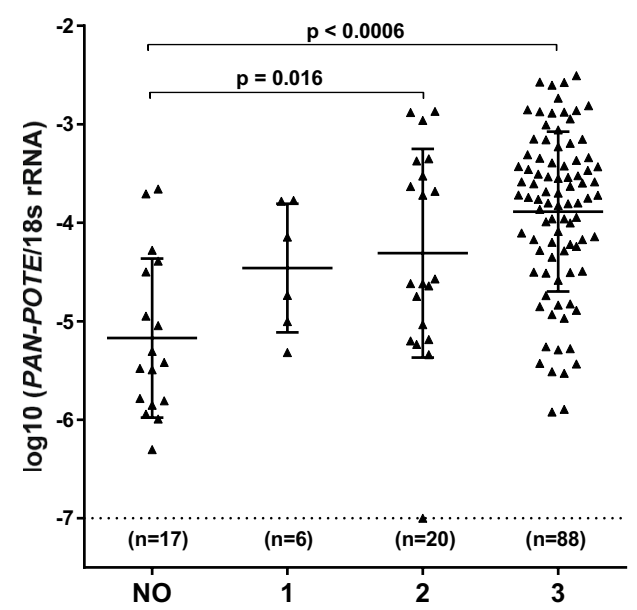

b

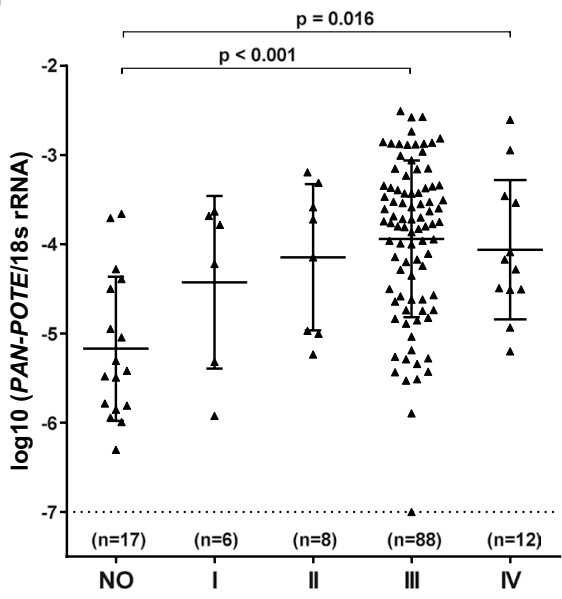

d

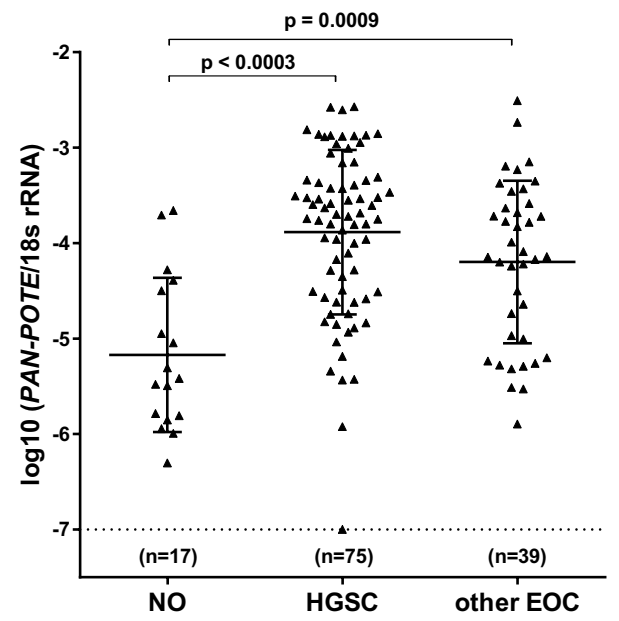

Figure 1. Pan-POTE expression in NO and EOC tissues. (a) NO and EOC. (b) NO and EOC separated by stage. (c) NO and EOC separated by grade. (d) NO and EOC separated into HGSC (serous histology, grade 2/3) and other EOC. Graphs show median values, and two-tailed Mann-Whitney tests with significant differences, after performing Bonferroni correction, are shown. Samples with no detectable Pan-POTE expression were plotted at $\log 10(-7)$ for clarity.

POTE expression in HGSC. HGSC frequently originates from precursor lesions in the fallopian tube epithelia (FTE), and the TCGA ovarian cancer project specifically focused on $\mathrm{HGSC}^{2,25,26}$. To focus our studies of POTEs on HGSC, and to examine individual POTEs using RNA-seq, we used Toil analyses ${ }^{23}$. As a control for HGSC, we combined normal tissue GTEx data from both ovary and fallopian tube (FT), as utilization of FT alone was not feasible due to limited sample size $(n=5)$, and because unseparated FT is only an approximation of FTE. This analysis revealed significant overexpression of 10/13 POTEs in HGSC (Fig. 2a-c). Amongst Groups 1 \& 2 POTEs, $A, B 2$, and $C$ showed significant upregulation, along with generally low or absent expression in control tissues (Fig. 2a). All Group 3 POTE-actin genes showed altered expression in HGSC, with all but one (POTEJ) being upregulated (Fig. 2b). We noted that POTE-actin expression was significantly upregulated in HGSC despite expression in the control tissues. Other Group 3 POTEs (POTEs $G / H / M$ ) were also highly upregulated in HGSC, but showed lower expression in control tissues than POTE-actin genes (Fig. 2c). Comparison of the expression of all POTEs revealed that POTEs $C, E, F$, and I show highest overall expression in HGSC (Fig. 2d).

We used unsupervised hierarchical clustering to compare POTE expression in TCGA HGSC data. POTEs generally clustered into three expression sub-groups: i) Groups 1 \& 2, ii) Group 3 POTE-actin genes, and iii) POTEs $G / H / M$ (Fig. 3a). We also identified different tumor clusters characterized by specific POTE expression patterns (Fig. 3a, right labels), and the most prominent clusters were characterized by high expression of POTEC and/or $P O T E$-actin genes. We conducted Spearman rank correlation testing of POTE expression, which confirmed that the three aforementioned POTE subgroups show correlated expression (Fig. 3b). In agreement with earlier data, the two POTEs that did not correlate within their respective subgroups (POTEs D and $J$ ) either showed very low expression in HGSC or were downregulated in HGSC compared to normal controls (Fig. 2a,b). 
a

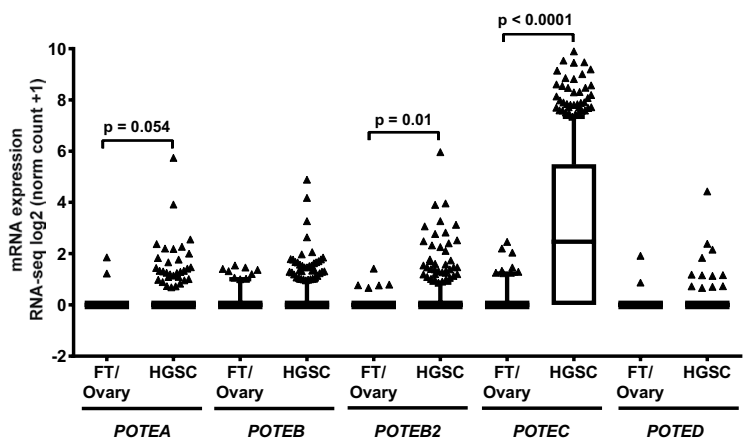

b
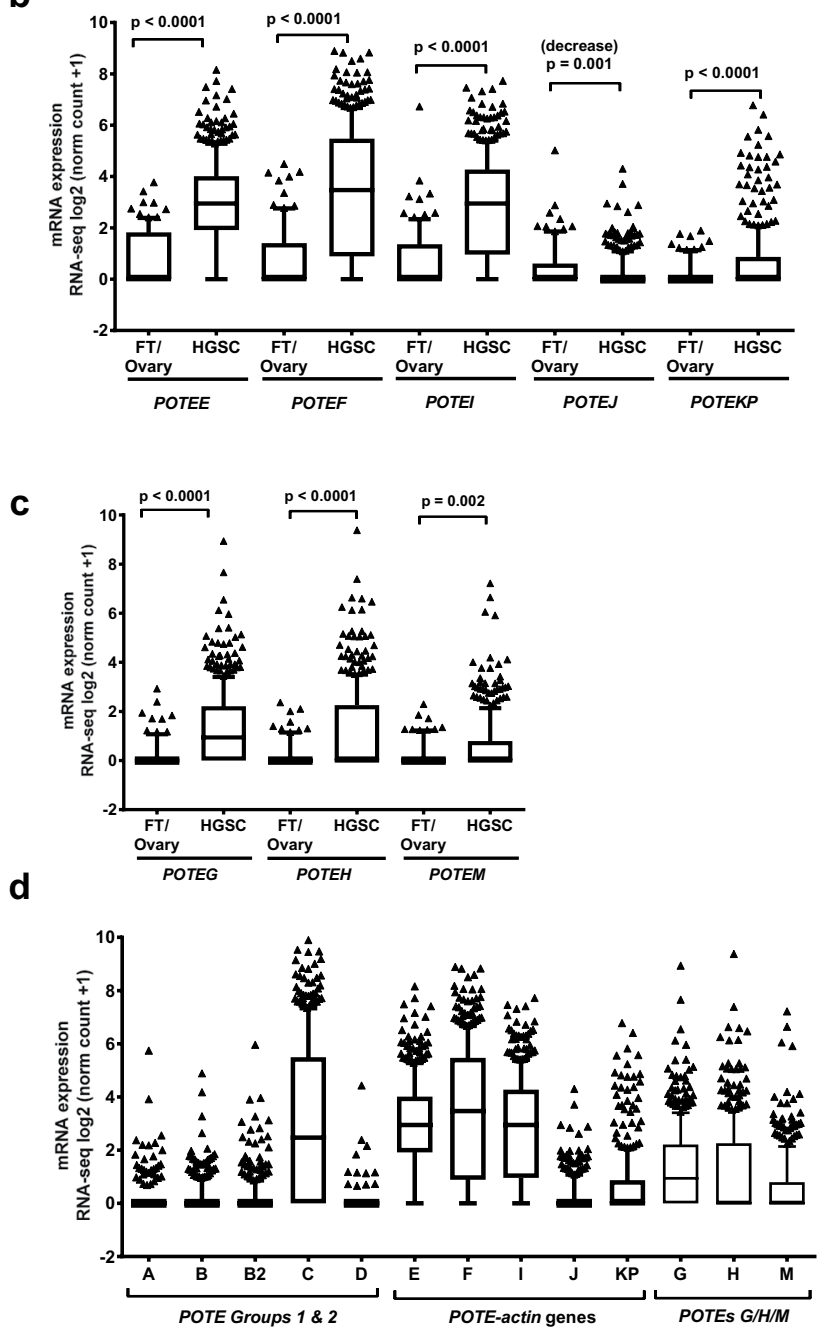

Figure 2. POTE expression in fallopian tube (FT) + ovary and HGSC. (a-c) Comparisons of POTE expression in normal controls $(\mathrm{n}=93)$ vs. HGSC $(\mathrm{n}=419)$. (a) Groups 1 \& 2 POTEs. (b) Group 3 POTE-actin genes. (c) Other Group 3 POTEs (i.e. POTEs $G / H / M$ ). (d) Comparison of POTE gene expression in HGSC. Box and whiskers plot, with medians, 10-90\%iles, and ranges indicated. Two-tailed Mann-Whitney tests with significant differences are shown.

HGSC patients often develop recurrent chemoresistant disease ${ }^{3}$. We compared POTE expression in patient-matched primary and recurrent HGSC using two independent RNA-seq data sets ${ }^{27,28}$. Data from Patch et al., showed altered expression of several POTEs, and identified POTEF, $I$, and $\mathrm{M}$ with significant upregulation in recurrent HGSC, both in individual patients and overall (Fig. 4a,c). In addition, POTEs C and E were upregulated in several patients. Data from Kreuzinger revealed a similar pattern of altered POTE expression, with increased expression of POTEs $C, F, I$, and $\mathrm{M}$ in recurrent HGSC. Only POTEC was significantly upregulated over the entire 


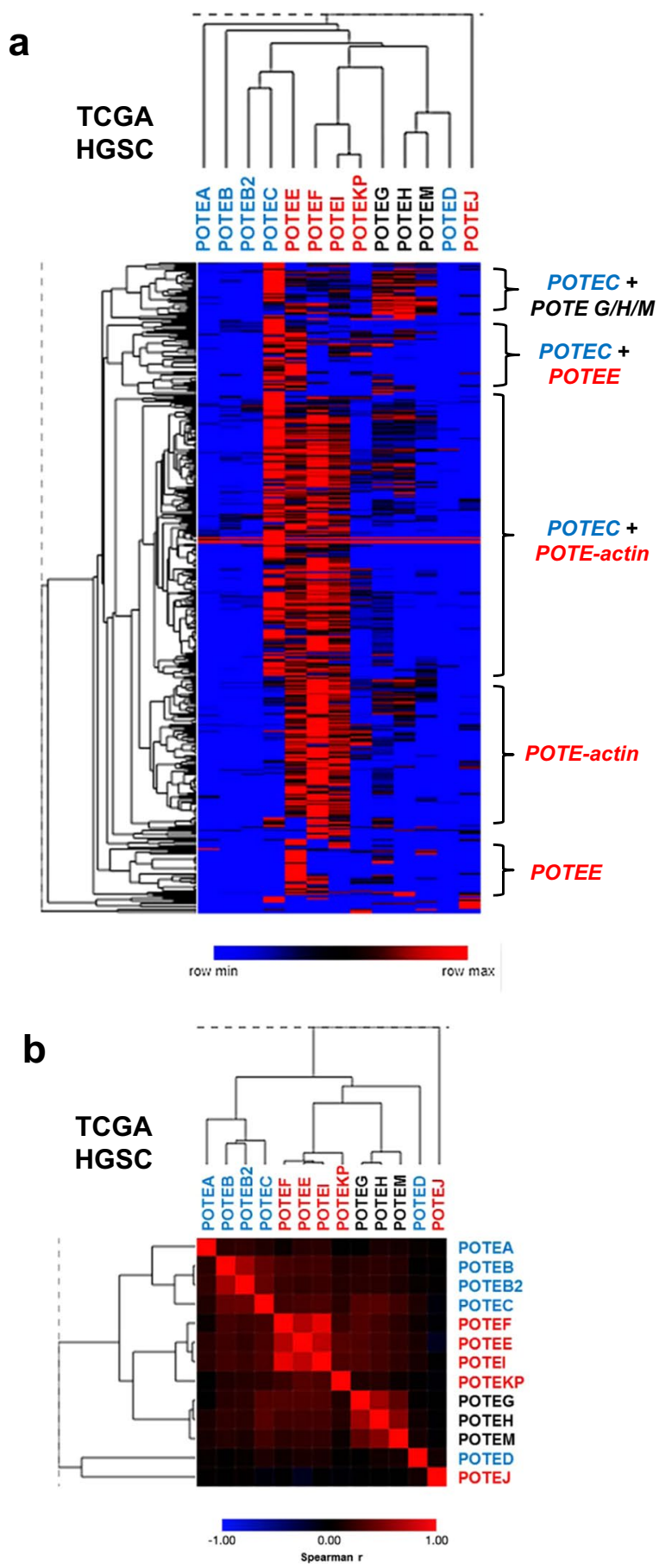

Figure 3. POTE expression in HGSC. (a) Expression heatmap of POTEs in TCGA HGSC data. Toil $\log 2$ normalized read counts shown, and coloring indicates row min to row max (see key). Samples showing enrichment for specific POTE expression patterns are labelled at right. (b) Spearman rank correlation matrix heatmap of POTE gene expression in TCGA HGSC. In both panels, POTE font color indicates POTE group: Groups 1 \& 2 (blue), Group 3 POTE-actin (red), POTE G/H/M (black).

patient population (Fig. 4b,d). Upregulated POTEs included at least one member of each previously identified POTE expression subgroup (i.e. Groups 1 \& 2, POTE-actin genes, and POTE $G, H, M$ ).

POTE expression and overall survival (OS) in EOC and HGSC. We tested the association of Pan-POTE expression with OS in EOC. Consistent with the observed increase of Pan-POTE with stage, grade, and HGSC (Fig. 1b-d), Pan-POTE associated with reduced OS in a univariate analysis, but not in a multivariate analysis (Fig. 5a; data not shown). We next tested the association of individual POTEs with OS using HGSC TCGA data, and observed that POTEE associated with reduced OS, using either two or three expression sub-groups (Fig. 5b,c). Consistently, POTEE was upregulated in HGSC compared to normal controls, showed heterogeneous 

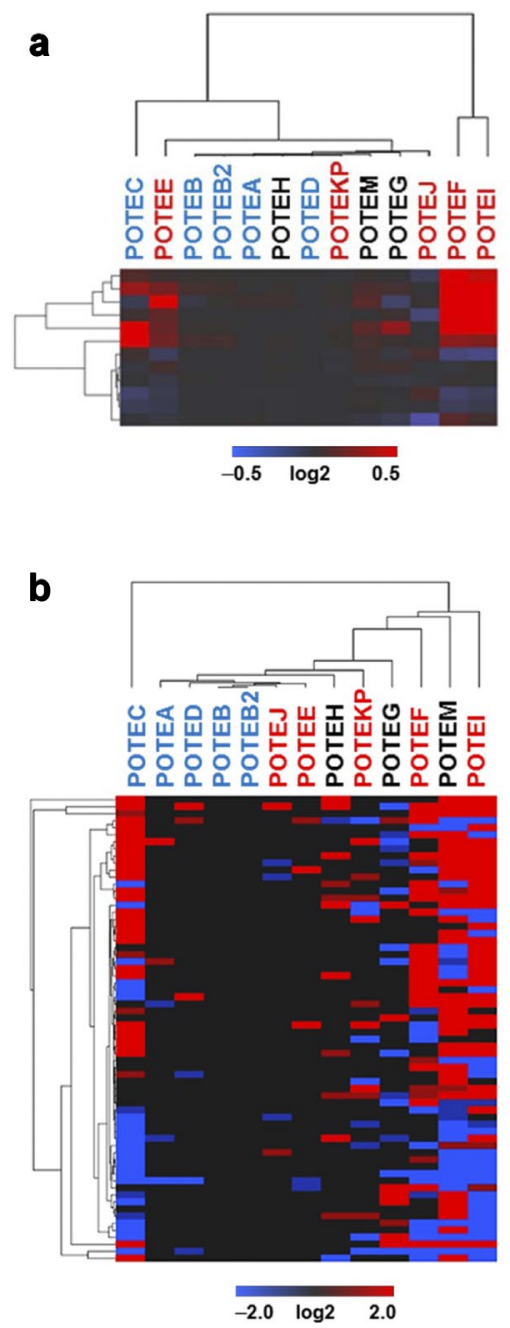

d
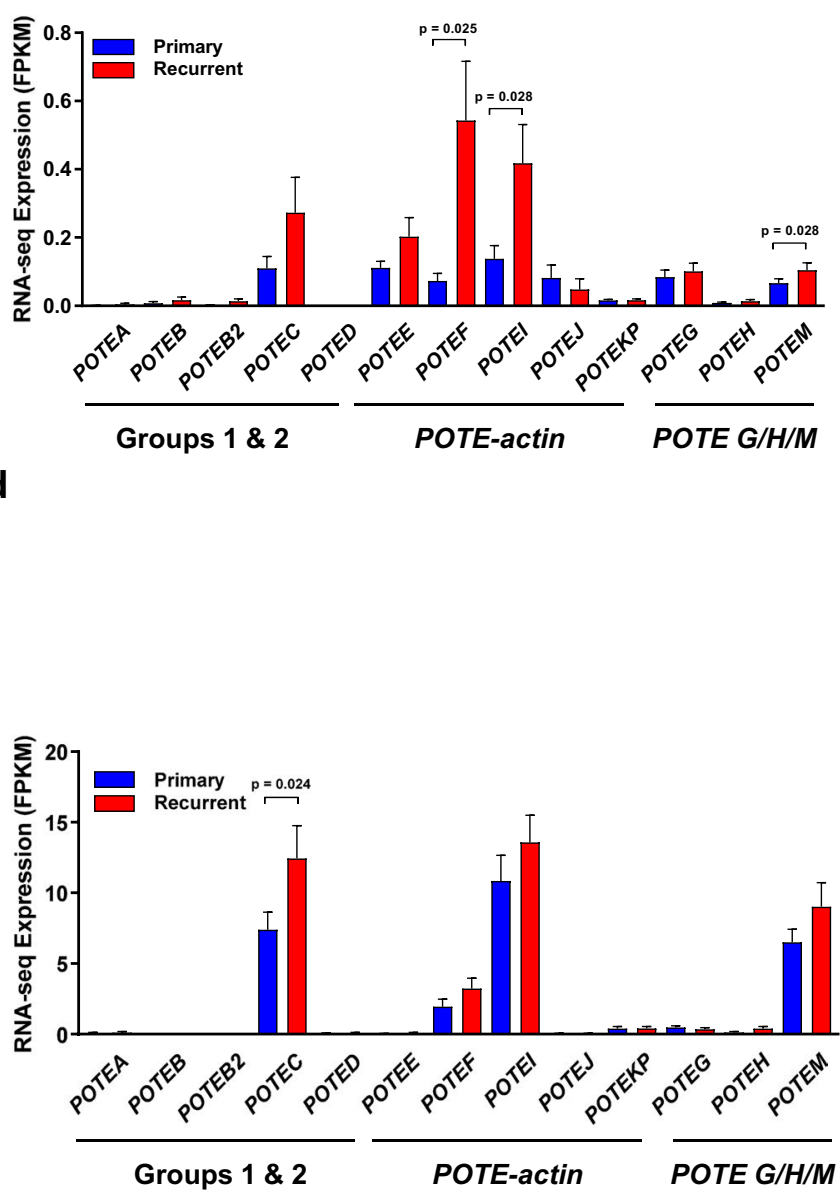

Figure 4. POTE expression in patient-matched primary and recurrent HGSC. (a,b) Expression heatmaps showing $\log 2$ fold changes for recurrent/primary HGSC. (c,d) POTE expression averages. (a,c) Data from Patch et al. ${ }^{27}$ ( $\mathrm{n}=12$ pairs). (b,d) Data from Kreuzinger et al. ${ }^{28}(\mathrm{n}=66$ pairs). Font color indicates POTE group: Groups 1 \& 2 (blue), Group 3 POTE-actin (red), other Group 3 (black). (c) Data from ${ }^{27}$ ( $\mathrm{n}=12$ pairs). (d) Data from $^{28}(\mathrm{n}=66$ pairs). Bars plot means + SEM; two-tailed student's t-test with significant differences are shown. In panels (a,b) POTE font color indicates POTE group: Groups 1 and 2 (blue), Group 3 POTE-actin (red), POTE $G / H / M$ (black).

expression in HGSC, and select patients showed increased POTEE expression at recurrence (Figs 2b, 3a, 4a,b). Other POTEs were not associated with HGSC OS (data not shown).

POTE expression in ovarian cancer cell lines. Cancer cell lines are valuable tools for functional studies $^{29}$. We measured Pan-POTE in a panel of cell lines relevant to EOC and HGSC, including cancer cell lines, and normal and immortalized ovarian surface epithelia (OSE) and FTE cells (Supplementary Table S2). Consistent with primary tumor data, Pan-POTE expression was significantly increased in ovarian cancer cells compared to control cells (Fig. 6a). Next, we examined the pattern of expression of individual POTEs in a large panel of ovarian cancer cell lines, using data from the cancer cell line encyclopedia (CCLE) ${ }^{24}$. POTE expression in CCLE ovarian lines segregated into the three POTE sub-groups described above (Fig. 6b,c). A large proportion of cell lines had elevated expression of POTE-actin genes (Fig. 6b).

POTE expression in pan-cancer TCGA and CCLE data. We utilized in silico resources to conduct an initial examination of POTE expression in pan-cancer ${ }^{24,30}$. Pan-cancer TCGA data showed similar POTE expression sub-groups and sample clusters as observed in HGSC (Fig. 7a,b). However, although the data were overall similar to HGSC, there were distinctions, including elevated POTEJ expression in a sub-set of tumors (Fig. 7a). In pan-cancer CCLE data, again similar POTE expression patterns were apparent, including sample clusters with increased expression of POTEC, POTE-actin genes, and Group 3 POTEs (Fig. 8a). Moreover, the three previously identified POTE expression sub-groups (Group $1 \& 2$, POTE-actin genes, and POTE G/H/M) perfectly segregated in pan-cancer CCLE data (Fig. 8a,b). 
a

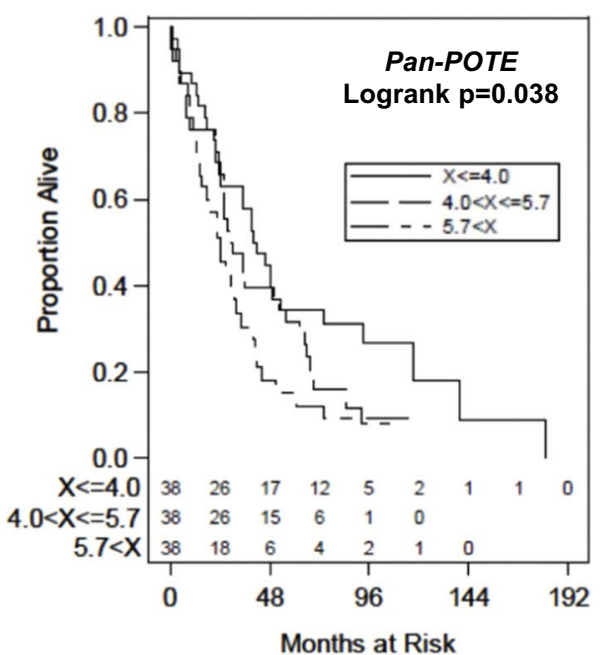

b

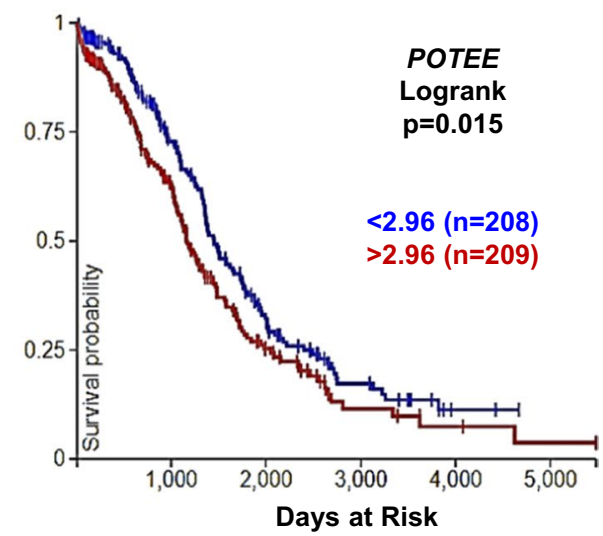

C

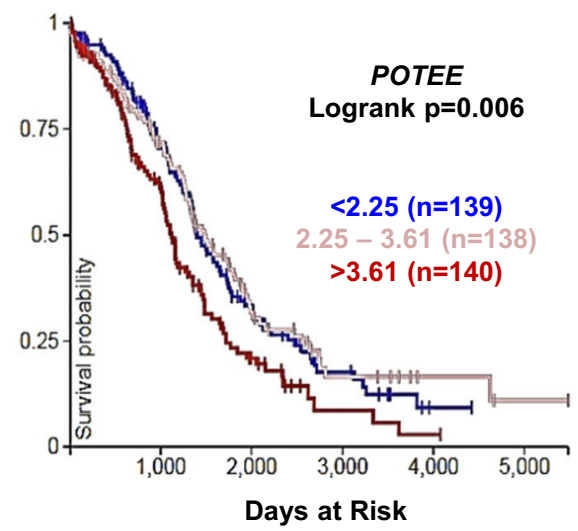

Figure 5. POTE expression and overall survival (OS) in EOC and HGSC. (a) Pan-POTE expression and OS in EOC $(\mathrm{n}=114)$. $(\mathbf{b}, \mathbf{c})$ POTEE expression and OS in TCGA HGSC $(\mathrm{n}=417)$, using either two $(\mathbf{b})$ or three $(\mathbf{c})$ expression subgroups.

\section{Discussion}

Pan-POTE expression is frequent in EOC and correlates with increased stage and grade, HGSC, and reduced OS. Although these data are valuable, it is important to determine individual POTE gene expression in the context of normal tissues and cancer. Due to extensive sequence homology this previously was difficult, requiring PCR cloning and Sanger sequencing ${ }^{21}$. To overcome this limitation, we utilized microarrays and, more extensively, RNA-seq. Microarray studies indicated that POTE sub-groups have increased expression in EOC compared to NO. Due to the availability of extensive RNA-seq data for $\mathrm{HGSC}^{2}$, and given our observation of Pan-POTE 
a

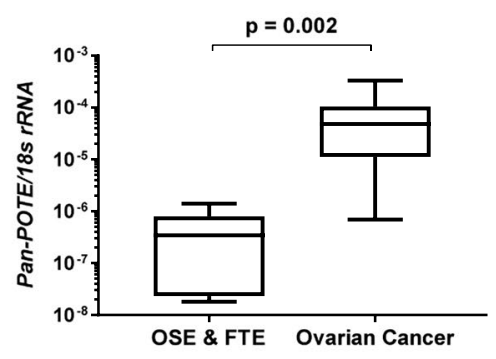

C

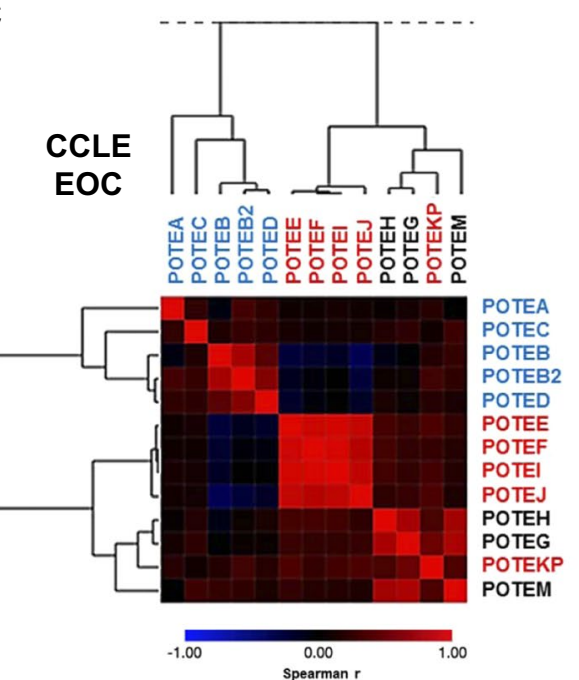

b

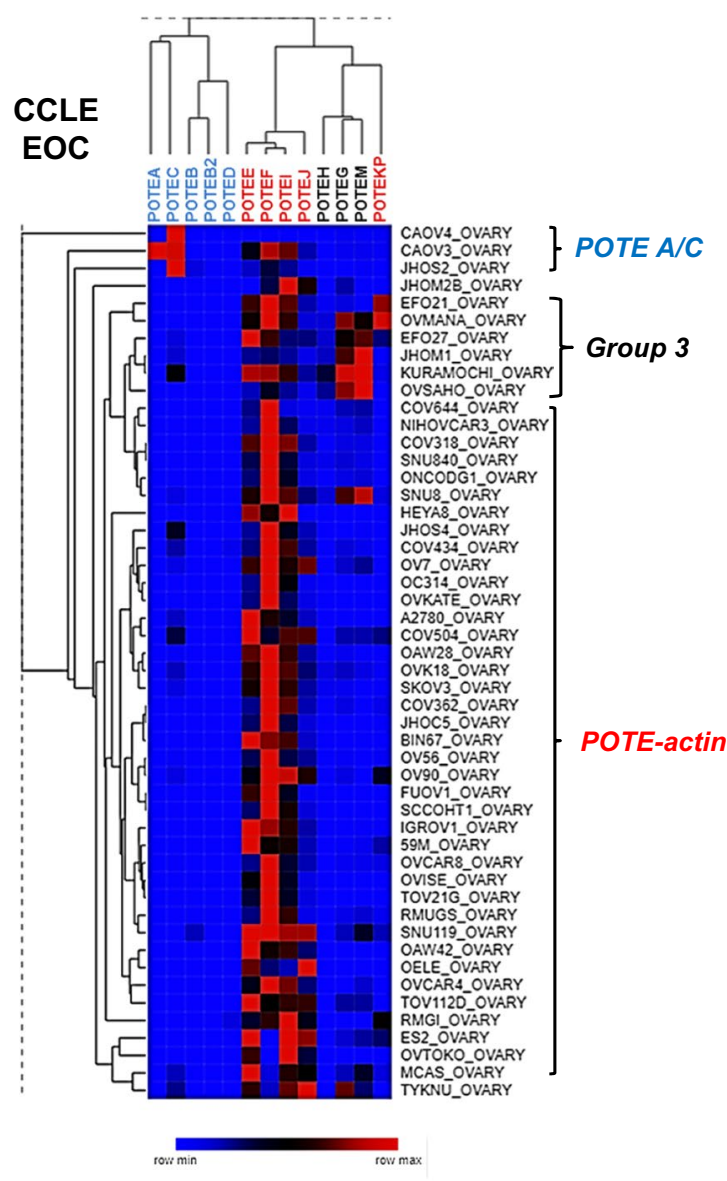

Figure 6. POTE expression in ovarian cancer and control cell lines. (a) Pan-POTE expression in control cells (ovarian surface epithelia, OSE; fallopian tube epithelia; FTE) and ovarian cancer cell lines. See Supplementary Table S1 for list of cell lines utiized. Box and whiskers plot, with medians, 25-75\%iles, and ranges indicated. Two-tailed Mann-Whitney test result shown. (b) POTE expression RNA-seq read counts in CCLE ovarian cancer cell lines $(n=50)$. Cell line names are shown, and samples showing enrichment for specific POTE expression patterns are labelled at right. (c) Spearman rank correlation matrix heatmap of POTE gene expression in CCLE ovarian cancer cell lines. In panels $(\mathbf{b}, \mathbf{c})$ POTE font color indicates POTE group: Groups 1 \& 2 (blue), Group 3 POTE-actin (red), POTE G/H/M (black).

overexpression in this EOC subtype, we focused subsequent studies on HGSC. We used TCGA HGSC data, and GTEx normal FT and ovary as the control, and our analyses revealed that most individual POTEs (10/13 genes) are overexpressed in HGSC. Importantly, GTEx data revealed that Groups 1 \& 2, but not Group 3, POTEs show a testis-specific expression pattern characteristic of CTAs. We conclude that Groups 1 \& 2 POTEs are CTAs that can be overexpressed in HGSC (3/5 genes), with POTEC showing the most robust overexpression. In contrast, Group 3 POTEs are not CTAs but are more commonly overexpressed in HGSC (7/8 genes), with POTEJ the lone exception. A caveat to our analysis is that GTEx used bulk tissues, not specifically isolated epithelial cells ${ }^{22}$. Because FTE secretory cells are the progenitor cell for HGSC, future studies should determine POTE expression in this cell type, as well as in HGSC precursor lesions in the distal $\mathrm{FT}^{25,26,31}$. Additionally, a recent study suggests that evaluation of testis-specific expression in the context of CTA gene classification benefits from the use of isolated testicular germ cells ${ }^{15}$.

POTEs showed patterns of correlated gene expression, and the three sub-groups were: i) Groups 1 \& 2 POTEs, ii) Group 3 POTE-actin genes, and iii) other Group 3 POTEs (i.e. POTEs G/H/M). These data suggest transcriptional co-regulation with sub-groups and divergence between groups. As CTA genes are regulated by epigenetic mechanisms ${ }^{9}$, it becomes relevant to determine whether epigenetics states, and/or specific transcription factors, explain the observed POTE expression sub-groups.

In addition to ovarian cancer, we conducted an initial examination of POTE expression in pan-cancer data sets from TCGA and CCLE. The data showed relative similarity of POTE expression patterns in pan-cancer. For example, sample sub-groups showed high enrichment of POTE-actin genes, POTEC, and Group 3 POTEs. Additionally, the three HGSC expression sub-groups were also apparent in pan-cancer data. Moving forward, it now becomes relevant to determine whether specific tumor types or lineages are enriched for specific patterns of POTE expression. 
a
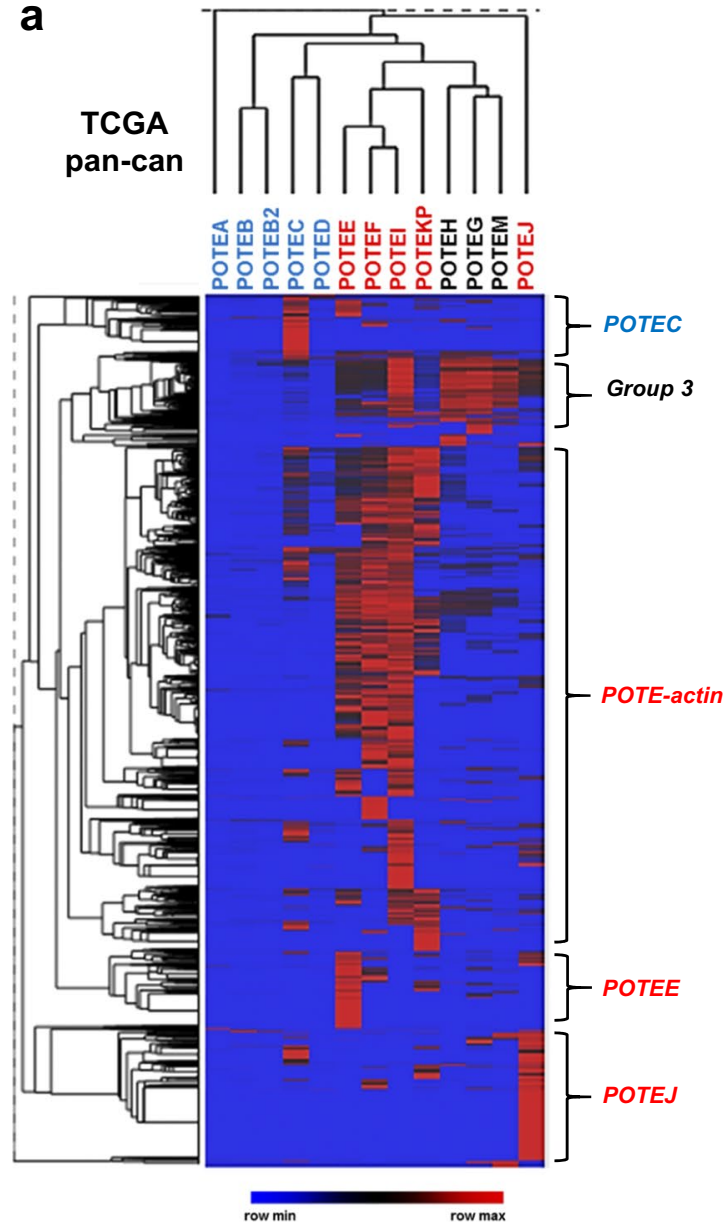

b

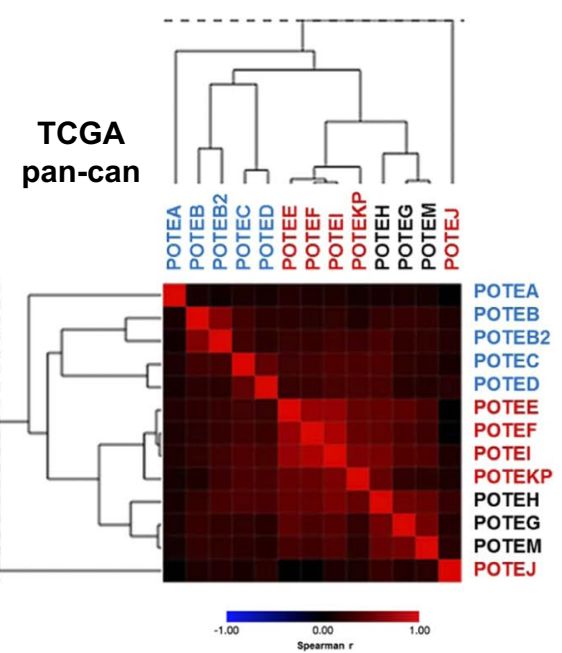

Figure 7. POTE expression in TCGA pan-cancer tissues $(\mathrm{n}=9345)$, determined using RNA-seq data from the UCSC Xena browser Toil. (a) Unsupervised hierarchical clustering of individual POTEs and pan-cancer cases. $\log 2$ normalized read counts are shown. Samples showing enrichment for specific POTE expression patterns are labelled at right. (b) Spearman rank correlation matrix heatmap of POTE gene expression in TCGA pan-cancer data. POTE font color indicates POTE group: Groups 1 \& 2 (blue), Group 3 POTE-actin (red), POTE G/H/M (black).

In contrast to POTE gene expression, POTE protein expression data in large cancer data sets is currently unavailable. In addition, commercial POTE antibodies recognize all or most POTEs, restricting their utility (data not shown). Supporting the relevance of our mRNA expression data, our prior studies of CTAs in EOC, 
a

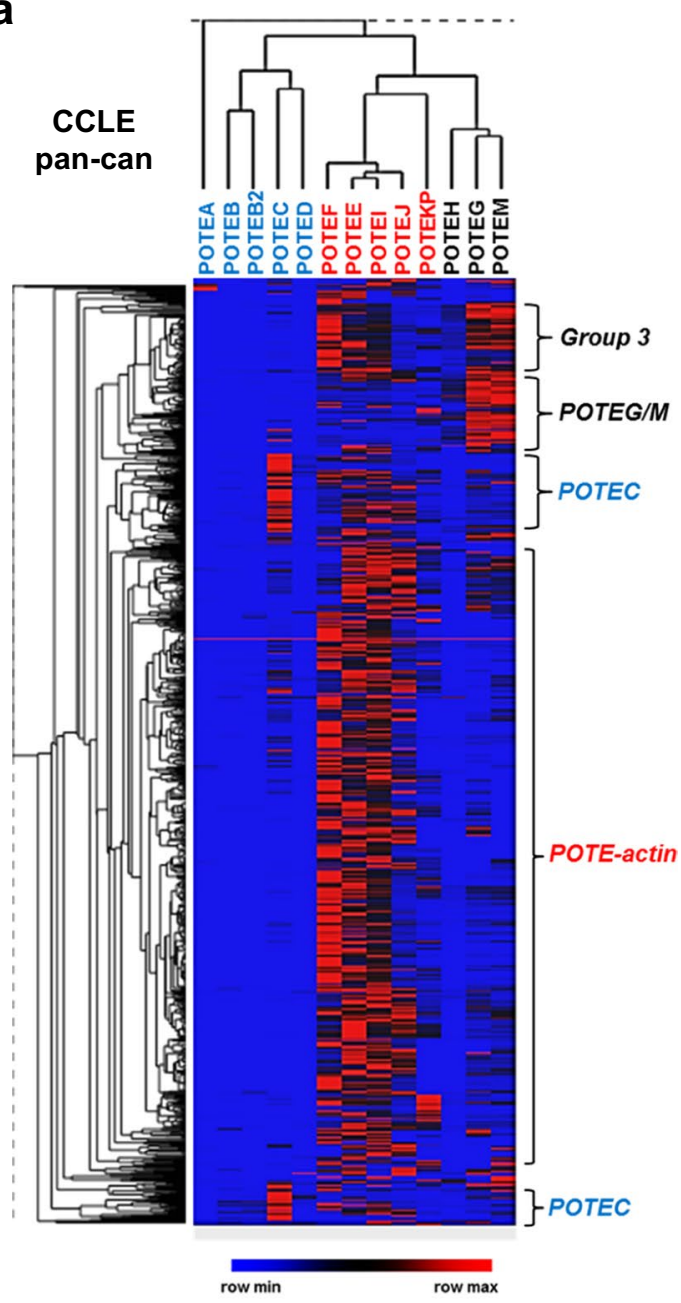

b

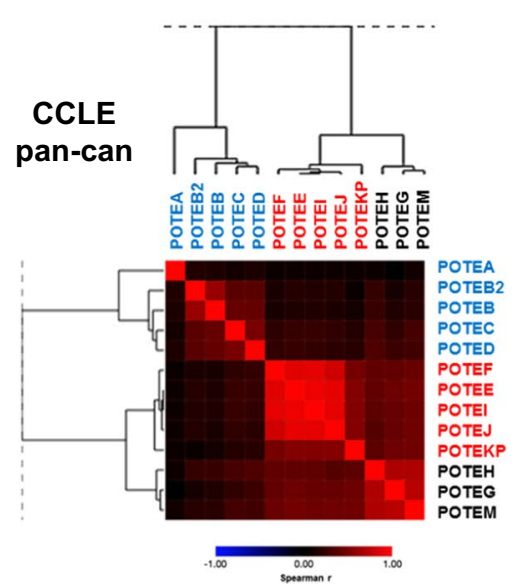

Figure 8. POTE expression in Cancer Cell Line Encyclopedia (CCLE) pan-cancer data. (a) POTE expression read counts in CCLE pan-cancer cell lines $(n=1076)$. Samples showing enrichment for specific POTE expression patterns are labelled at right. (b) Spearman rank correlation matrix heatmap of POTE gene expression in CCLE pan-cancer cell lines. POTE font color indicates POTE group: Groups 1 \& 2 (blue), Group 3 POTE-actin (red), POTE G/H/M (black).

including CTCFL (BORIS), CT45, and PRAME, revealed significant correlations between mRNA and protein expression $^{32-34}$. Nevertheless, an important goal is to measure POTE protein expression levels in EOC and HGSC and to determine the relationship of protein expression to clinicopathology. Of note, a recent proteomic study reported increased POTEE expression in breast cancer $^{35}$. It is intriguing that we observed that POTEE was the 
only POTE gene associated with reduced OS in HGSC, given the fact that HGSC has high genomic similarity to basal breast cancer ${ }^{36}$.

POTE protein expression was previously detected in human testis and spermatids, where it was associated with apoptosis ${ }^{37,38}$. Moreover, studies of cancer cells provide tentative support of a role for POTEs in apoptosis ${ }^{39,40}$. In addition, POTE-actin proteins appear likely to play a role in cytoskeletal function given their structure. Future work on POTE function in ovarian and other cancers might thus focus on apoptosis and cytoskeletal functions as starting points for investigation.

For functional cancer studies, cell lines are an invaluable tool. In this context, we observed that EOC/HGSC cell lines have significantly elevated POTE expression compared to normal OSE and FTE controls. In particular, POTE expression in the CCLE cell lines provides useful insight into model choice to study of POTE function in ovarian and other cancers.

The fact that several POTEs are not CTAs, combined with the high conservation of POTE proteins, could make immunological approaches to target POTEs difficult, despite the fact that POTE epitopes are capable of generating human CTL responses ${ }^{41}$. Regardless of the limitations in immunological targeting of POTEs, frequent POTE overexpression in EOC, HGSC, and other cancers, along with limited or absent expression in most normal tissues, supports POTEs as potential therapeutic targets. An important next step will be to determine whether (and which) POTEs have oncogenic function. Such data will provide insight into the potential of POTE-targeted approaches for cancer treatment.

\section{Methods}

POTE expression in human adult normal tissues. We determined the expression of individual POTEs in human adult normal tissues using GTEx ${ }^{22}$. We obtained GTEx RNAseq data using GeneCards (http://www. genecards.org/).

Pan-POTE expression in human EOC and normal ovary (NO) tissues. We obtained fresh-frozen human EOC and bulk normal ovary (NO; obtained from patients without malignancy). All samples were collected using IRB-approved protocols at Roswell Park Comprehensive Cancer Center (RPCCC) ${ }^{42}$. All experiments using human samples were approved by the Institutional Review Board of the RPCCC and the Institutional Review Board of the University of Nebraska Medical Center (UNMC), and all methods were performed in accordance with relevant guidelines and regulations. Informed consent was obtained from all subjects and all subjects were over the age of 18 . We processed tissues as described ${ }^{43}$. We extracted RNA using TRIzol (Invitrogen) and synthesized cDNA using iScript cDNA Synthesis Kit (BioRad). We performed qPCR using the BioRad CFX Connect system with SYBR green master mix (Qiagen), and primers from IDT. We amplified Pan-POTE (i.e. all POTE genes) as described ${ }^{19}$. We also determined POTE expression in EOC $(\mathrm{n}=40)$ and NO $(\mathrm{n}=3)$ using Affymetrix HG 1.0ST arrays, performed by the University at Buffalo Center of Excellence in Bioinformatics and Life Sciences (UBCOE). We normalized microarray probe cell intensity data (.cel) using the Affymetrix Expression Console (version 1.3.0.187) software running the Robust Multi-chip Averaging (RMA) background correction and quantile normalization using a linear scale.

POTE expression in fallopian tube (FT), ovary, and HGSC tissues. We obtained Toil GTEx data for FT and ovary, and Toil TCGA data for HGSC and pan-cancer. All data correspond to RNA-seq normalized read counts. We obtained data from the UCSC Xena Browser (https://xenabrowser.net) ${ }^{23}$.

POTE expression in primary and recurrent HGSC. We obtained POTE RNA-seq data from patient-matched primary and recurrent HGSC using the European Genome-phenome Archive (EGA) https:// ega-archive.org/. We analyzed EGAD00001000877 ( $\mathrm{n}=12$ pairs) and EGAD00010001403 $(\mathrm{n}=66 \text { pairs })^{27,28}$.

POTE expression and overall survival (OS) in EOC and HGSC. For EOC, we defined overall survival (OS) as the time between the date of diagnosis and death, and censored patients who were alive at the time of analysis at the date of last follow up. We split EOC patients into Pan-POTE expression tertiles and compared OS using Kaplan-Meier analysis and Logrank test. For HGSC, we analyzed individual POTE expression vs. HGSC survival using the UCSC Xena Browser (https://xenabrowser.net).

POTE expression in ovarian cancer, OSE, and FTE cells. We measured Pan-POTE expression as described above ${ }^{19}$. We obtained OVCAR3, A2780, and OVCAR429 from ATCC and cultured as described ${ }^{43}$. We obtained and cultured Kuramochi, OVSAHO, SNU119, COV318, COV362, OVCAR4, and SV40 large T-antigen immortalized normal human OSE (IOSE-SV) cells as described ${ }^{44}$. We obtained SKOV3 from ATCC and cultured in McCoy's media with standard supplementation. We obtained primary human OSE from ScienCell and cultured according to manufacturers' instructions. We obtained CAOV3 and OVCAR5 from Dr. Anirban Mitra and cultured as described ${ }^{45}$. We obtained OVCAR8 cells from the NCI and cultured in DMEM, using standard supplementation. We obtained EFO-21 from the MD Anderson Cancer Center (MDACC) Cell Line Core and cultured in RPMI 1640 and 20\% FBS with standard supplementation. We obtained FU-OV1 from MDACC Cell Line Core and cultured in DMEM/F12 with standard supplementation. We obtained and cultured FT190, FT237, FT282, and FT282-CCNE1 as described ${ }^{31,46,47}$. We generated a clonal FT282 cell line, FT282-c11, and FT282-c11-FOXM1c cells as described in Supplementary Methods. We obtained CCLE RNA-seq data (normalized read counts, release date: May 2, 2018), generated and funded by Broad Cancer Dependency Map (https:// depmap.org/broad/), using the Broad CCLE Portal (https://portals.broadinstitute.org/ccle/data). We analyzed data for both ovarian cancer cell lines in $\operatorname{CCLE}(\mathbf{n}=\mathbf{5 0})$ and pan-cancer cell lines $(\mathbf{n}=\mathbf{1 0 7 6})$. 
Statistical analyses. We used descriptive statistics as described in the individual figure legends to compare group differences. We used Spearman rank order tests to measure expression correlations. We assigned $\mathrm{p}<0.05$ as the cutoff for statistical significance. We used GraphPad Prism to conduct statistical analyses. Statistical analyses relevant to survival are described above.

\section{Data Availability}

The datasets generated during and/or analyzed during the current study are available from the corresponding author on reasonable request.

\section{References}

1. Karst, A. M. \& Drapkin, R. Ovarian cancer pathogenesis: a model in evolution. J Oncol 2010, 932371, https://doi.org/10.1155/2010/932371 (2010).

2. Integrated genomic analyses of ovarian carcinoma. Nature 474, 609-615, https://doi.org/10.1038/nature10166 (2011).

3. Bowtell, D. D. et al. Rethinking ovarian cancer II: reducing mortality from high-grade serous ovarian cancer. Nat Rev Cancer $\mathbf{1 5}$, 668-679, https://doi.org/10.1038/nrc4019 (2015).

4. Chen, Y. \& Du, H. The promising PARP inhibitors in ovarian cancer therapy: From Olaparib to others. Biomed Pharmacother 99 , 552-560, https://doi.org/10.1016/j.biopha.2018.01.094 (2018).

5. Lord, C. J. \& Ashworth, A. PARP inhibitors: Synthetic lethality in the clinic. Science 355, 1152-1158, https://doi.org/10.1126/science. aam7344 (2017).

6. Konstantinopoulos, P. A. \& Matulonis, U. A. PARP inhibitors in ovarian cancer: a trailblazing and transformative journey. Clin Cancer Res, https://doi.org/10.1158/1078-0432.CCR-18-1314 (2018).

7. Simpson, A. J., Caballero, O. L., Jungbluth, A., Chen, Y. T. \& Old, L. J. Cancer/testis antigens, gametogenesis and cancer. Nat Rev Cancer 5, 615-625, https://doi.org/10.1038/nrc1669 (2005).

8. Coulie, P. G., V den Eynde, B. J., van der Bruggen, P. \& Boon, T. Tumour antigens recognized by T lymphocytes: at the core of cancer immunotherapy. Nat Rev Cancer 14, 135-146, https://doi.org/10.1038/nrc3670 (2014).

9. Akers, S. N., Odunsi, K. \& Karpf, A. R. Regulation of cancer germline antigen gene expression: implications for cancer immunotherapy. Future Oncol 6, 717-732 (2010).

10. De Smet, C. \& Loriot, A. DNA hypomethylation and activation of germline-specific genes in cancer. Adv Exp Med Biol 754, 149-166, https://doi.org/10.1007/978-1-4419-9967-2_7 (2013).

11. Want, M. Y., Lugade, A. A., Battaglia, S. \& Odunsi, K. Nature of tumor rejection antigens in ovarian cancer. Immunology, https://doi. org/10.1111/imm.12951 (2018).

12. Whitehurst, A. W. Cause and consequence of cancer/testis antigen activation in cancer. Annu Rev Pharmacol Toxicol 54, 251-272, https://doi.org/10.1146/annurev-pharmtox-011112-140326 (2014).

13. Wang, C. et al. Systematic identification of genes with a cancer-testis expression pattern in 19 cancer types. Nat Commun 7, 10499, https://doi.org/10.1038/ncomms10499 (2016).

14. Maxfield, K. E. et al. Comprehensive functional characterization of cancer-testis antigens defines obligate participation in multiple hallmarks of cancer. Nat Commun 6, 8840, https://doi.org/10.1038/ncomms9840 (2015)

15. Bruggeman, J. W., Koster, J., Lodder, P., Repping, S. \& Hamer, G. Massive expression of germ cell-specific genes is a hallmark of cancer and a potential target for novel treatment development. Oncogene, https://doi.org/10.1038/s41388-018-0357-2 (2018).

16. Bera, T. K. et al. POTE, a highly homologous gene family located on numerous chromosomes and expressed in prostate, ovary, testis, placenta, and prostate cancer. Proc Natl Acad Sci USA 99, 16975-16980, https://doi.org/10.1073/pnas.262655399 (2002).

17. Hahn, Y., Bera, T. K., Pastan, I. H. \& Lee, B. Duplication and extensive remodeling shaped POTE family genes encoding proteins containing ankyrin repeat and coiled coil domains. Gene 366, 238-245, https://doi.org/10.1016/j.gene.2005.07.045 (2006).

18. Bera, T. K. et al. Selective POTE paralogs on chromosome 2 are expressed in human embryonic stem cells. Stem Cells Dev 17, 325-332, https://doi.org/10.1089/scd.2007.0079 (2008).

19. Lee, Y. et al. Evolution and expression of chimeric POTE-actin genes in the human genome. Proc Natl Acad Sci USA 103, 17885-17890, https://doi.org/10.1073/pnas.0608344103 (2006).

20. Bera, T. K. et al. Five POTE paralogs and their splice variants are expressed in human prostate and encode proteins of different lengths. Gene 337, 45-53, https://doi.org/10.1016/j.gene.2004.05.009 (2004).

21. Bera, T. K. et al. POTE paralogs are induced and differentially expressed in many cancers. Cancer Res 66, 52-56, https://doi. org/10.1158/0008-5472.CAN-05-3014 (2006).

22. Consortium, G. T. The Genotype-Tissue Expression (GTEx) project. Nat Genet 45, 580-585, https://doi.org/10.1038/ng.2653 (2013).

23. Vivian, J. et al. Toil enables reproducible, open source, big biomedical data analyses. Nat Biotechnol 35, 314-316, https://doi. org/10.1038/nbt.3772 (2017).

24. Barretina, J. et al. The Cancer Cell Line Encyclopedia enables predictive modelling of anticancer drug sensitivity. Nature 483, 603-607, https://doi.org/10.1038/nature11003 (2012).

25. Klinkebiel, D., Zhang, W., Akers, S. N., Odunsi, K. \& Karpf, A. R. DNA Methylome Analyses Implicate Fallopian Tube Epithelia as the Origin for High-Grade Serous Ovarian Cancer. Mol Cancer Res 14, 787-794, https://doi.org/10.1158/1541-7786.MCR-16-0097 (2016).

26. Labidi-Galy, S. I. et al. High grade serous ovarian carcinomas originate in the fallopian tube. Nat Commun 8, 1093, https://doi. org/10.1038/s41467-017-00962-1 (2017).

27. Patch, A. M. et al. Whole-genome characterization of chemoresistant ovarian cancer. Nature 521, 489-494, https://doi.org/10.1038/ nature14410 (2015).

28. Kreuzinger, C. et al. A Complex Network of Tumor Microenvironment in Human High-Grade Serous Ovarian Cancer. Clin Cancer Res 23, 7621-7632, https://doi.org/10.1158/1078-0432.CCR-17-1159 (2017).

29. Domcke, S., Sinha, R., Levine, D. A., Sander, C. \& Schultz, N. Evaluating cell lines as tumour models by comparison of genomic profiles. Nat Commun 4, 2126, https://doi.org/10.1038/ncomms3126 (2013).

30. Liu, J. et al. An Integrated TCGA Pan-Cancer Clinical Data Resource to Drive High-Quality Survival Outcome Analytics. Cell 173, 400-416 e411, https://doi.org/10.1016/j.cell.2018.02.052 (2018).

31. Perets, R. et al. Transformation of the fallopian tube secretory epithelium leads to high-grade serous ovarian cancer in Brca;Tp53;Pten models. Cancer Cell 24, 751-765, https://doi.org/10.1016/j.ccr.2013.10.013 (2013).

32. Woloszynska-Read, A. et al. Coordinated cancer germline antigen promoter and global DNA hypomethylation in ovarian cancer: association with the BORIS/CTCF expression ratio and advanced stage. Clin Cancer Res 17, 2170-2180, https://doi. org/10.1158/1078-0432.CCR-10-2315 (2011).

33. Zhang, W. et al. PRAME expression and promoter hypomethylation in epithelial ovarian cancer. Oncotarget 7, 45352-45369, https:// doi.org/10.18632/oncotarget.9977 (2016).

34. Zhang, W. et al. DNA hypomethylation-mediated activation of Cancer/Testis Antigen 45 (CT45) genes is associated with disease progression and reduced survival in epithelial ovarian cancer. Epigenetics 10, 736-748, https://doi.org/10.1080/15592294.2015.106 2206 (2015). 
35. Cine, N. et al. Identification of ApoA1, HPX and POTEE genes by omic analysis in breast cancer. Oncol Rep 32, 1078-1086, https:// doi.org/10.3892/or.2014.3277 (2014).

36. Cancer Genome Atlas, N. Comprehensive molecular portraits of human breast tumours. Nature 490, 61-70, https://doi.org/10.1038/ nature11412 (2012)

37. Bera, T. K., Walker, D. A., Sherins, R. J. \& Pastan, I. POTE protein, a cancer-testis antigen, is highly expressed in spermatids in human testis and is associated with apoptotic cells. Biochem Biophys Res Commun 417, 1271-1274, https://doi.org/10.1016/j. bbrc.2011.12.125 (2012).

38. Ise, T. et al. Expression of POTE protein in human testis detected by novel monoclonal antibodies. Biochem Biophys Res Commun 365, 603-608, https://doi.org/10.1016/j.bbrc.2007.10.195 (2008).

39. Redfield, S. M. et al. TheC-terminal common to group 3 POTES (CtG3P): a newly discovered nucleolar marker associated with malignant progression and metastasis. Am J Cancer Res 3, 278-289 (2013).

40. Liu, X. F., Bera, T. K., Liu, L. J. \& Pastan, I. A primate-specific POTE-actin fusion protein plays a role in apoptosis. Apoptosis 14, 1237-1244, https://doi.org/10.1007/s10495-009-0392-0 (2009).

41. Huang, Y. H. et al. Identification and enhancement of HLA-A2.1-restricted CTL epitopes in a new human cancer antigen-POTE. PLoS One 8, e64365, https://doi.org/10.1371/journal.pone.0064365 (2013).

42. Akers, S. N. et al. LINE1 and Alu repetitive element DNA methylation in tumors and white blood cells from epithelial ovarian cancer patients. Gynecol Oncol 132, 462-467, https://doi.org/10.1016/j.ygyno.2013.12.024 (2014).

43. Woloszynska-Read, A. et al. DNA methylation-dependent regulation of BORIS/CTCFL expression in ovarian cancer. Cancer Immun 7, 21 (2007).

44. Barger, C. J. et al. Genetic determinants of FOXM1 overexpression in epithelial ovarian cancer and functional contribution to cell cycle progression. Oncotarget 6, 27613-27627, https://doi.org/10.18632/oncotarget.4546 (2015).

45. Mitra, A. K. et al. In vivo tumor growth of high-grade serous ovarian cancer cell lines. Gynecol Oncol 138, 372-377, https://doi. org/10.1016/j.ygyno.2015.05.040 (2015).

46. Karst, A. M. \& Drapkin, R. Primary culture and immortalization of human fallopian tube secretory epithelial cells. Nat Protoc 7 , 1755-1764, https://doi.org/10.1038/nprot.2012.097 (2012).

47. Karst, A. M. et al. Cyclin E1 deregulation occurs early in secretory cell transformation to promote formation of fallopian tubederived high-grade serous ovarian cancers. Cancer Res 74, 1141-1152, https://doi.org/10.1158/0008-5472.CAN-13-2247 (2014).

\section{Acknowledgements}

We thank Nelly Auersperg, Francis Balkwill, and Anirban Mitra for generously providing cell lines, and the UBCOE (microarrays), RPCCC Bioinformatics, and UNMC Epigenomics Core facilities for support. This project was supported by NIH RO1CA116674, The Betty J. and Charles D. McKinsey Ovarian Cancer Research Fund, the FPBCC Support Grant (NIH P30CA036727), the RPCCC Support Grant (NIH P30CA016056), NIH T32CA009476, UNMC Program of Excellence Assistantship, and NIH F99CA212470.

\section{Author Contributions}

A.R.K. conceived and supervised the project, and wrote the manuscript. C.J.B., W.Z., A.S., L.C., S.R.J., C.N.K., A.M., J.M. and D.K. generated and/or analyzed data. R.D. and K.O. provide reagents. All authors approved the manuscript. C.J.B. and W.Z. contributed equally to this work.

\section{Additional Information}

Supplementary information accompanies this paper at https://doi.org/10.1038/s41598-018-35567-1.

Competing Interests: The authors declare no competing interests.

Publisher's note: Springer Nature remains neutral with regard to jurisdictional claims in published maps and institutional affiliations.

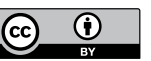

Open Access This article is licensed under a Creative Commons Attribution 4.0 International

License, which permits use, sharing, adaptation, distribution and reproduction in any medium or format, as long as you give appropriate credit to the original author(s) and the source, provide a link to the Creative Commons license, and indicate if changes were made. The images or other third party material in this article are included in the article's Creative Commons license, unless indicated otherwise in a credit line to the material. If material is not included in the article's Creative Commons license and your intended use is not permitted by statutory regulation or exceeds the permitted use, you will need to obtain permission directly from the copyright holder. To view a copy of this license, visit http://creativecommons.org/licenses/by/4.0/.

(C) The Author(s) 2018 